University of New Hampshire

University of New Hampshire Scholars' Repository

Space Science Center

Institute for the Study of Earth, Oceans, and

Space (EOS)

1994

\title{
Spectral properties of gamma-ray bursts observed by COMPTEL
}

\author{
L O. Hanlon \\ ESTEC \\ K Bennett \\ ESTEC \\ O R. Williams \\ ESTEC \\ C Winkler \\ ESTEC \\ W Collmar \\ Max-Planck-Institut für extraterrestriche Physik
}

See next page for additional authors

Follow this and additional works at: https://scholars.unh.edu/ssc

Part of the Astrophysics and Astronomy Commons

\section{Recommended Citation \\ Spectral properties of gamma-ray bursts observed by COMPTEL Hanlon, L. O. and Bennett, K. and Williams, O. R. and Winkler, C. and Collmar, W. and Diehl, R. and Greiner, J. and Schönfelder, V. and Steinle, H. and Strong, A. and Varendorff, M. and van Dijk, R. and den Herder, J. W. and Hermsen, W. and Kuiper, L. and Connors, A. and Kippen, R. M. and McConnell, M. and Ryan, J., AIP Conference Proceedings, 307, 275-279 (1994), DOl:http://dx.doi.org/10.1063/1.45809}

This Conference Proceeding is brought to you for free and open access by the Institute for the Study of Earth, Oceans, and Space (EOS) at University of New Hampshire Scholars' Repository. It has been accepted for inclusion in Space Science Center by an authorized administrator of University of New Hampshire Scholars' Repository. For more information, please contact Scholarly.Communication@unh.edu. 


\section{Authors}

L O. Hanlon, K Bennett, O R. Williams, C Winkler, W Collmar, R Diehl, J Greiner, V Schonfelder, H Steinle, A W. Strong, M Varendorff, R VanDijk, J W. den Herder, W Hermsen, L Kuiper, A Connors, R M. Kippen, Mark L. McConnell, and James M. Ryan 


\section{AIP | proceedings}

\section{Spectral properties of gammaray bursts observed by COMPTEL}

L. O. Hanlon, K. Bennett, O. R. Williams, C. Winkler, W. Collmar, R. Diehl, J. Greiner, V. Schönfelder, H. Steinle, A. Strong, M. Varendorff, R. van Dijk, J. W. den Herder, W. Hermsen, L. Kuiper, A. Connors, R. M. Kippen, M. McConnell, and J. Ryan

Citation: AIP Conference Proceedings 307, 275 (1994); doi: 10.1063/1.45809

View online: $\mathrm{http} / / / \mathrm{dx}$.doi.org/10.1063/1.45809

View Table of Contents:

http://scitation.aip.org/content/aip/proceeding/aipcp/307?ver=pdfcov

Published by the AIP Publishing

Articles you may be interested in

Spectral curvature in highenergy gamma ray bursts observed by the BATSE large area detectors

AIP Conf. Proc. 307, 318 (1994); 10.1063/1.45886

The energy emission of gammaray bursts and solar flares

AIP Conf. Proc. 307, 308 (1994); 10.1063/1.45884

Continuum evolution of bright gamma ray bursts observed by BATSE AIP Conf. Proc. 307, 298 (1994); 10.1063/1.45882

Comparison of BATSE, COMPTEL, EGRET, and OSSE spectra of GRB 910601 AIP Conf. Proc. 307, 283 (1994); 10.1063/1.45880

Observation of gammaray burst spectral at high energy by the Phebus experiment AIP Conf. Proc. 265, 43 (1991); 10.1063/1.42776 


\title{
SPECTRAL PROPERTIES OF GAMMA-RAY BURSTS OBSERVED BY COMPTEL
}

\author{
L.O. Hanlon, K. Bennett, O.R. Williams, C. Winkler \\ Astrophysics Division, ESTEC, NL-2200 AG Noordwijk, The Netherlands \\ W. Collmar, R. Diehl, J. Greiner, V. Schönfelder, \\ H. Steinle, A. Strong, M. Varendorff \\ Max-Planck-Institut für Extraterrestrische Physik, 85740 Garching, Germany \\ R. van Dijk, ${ }^{*}$ J.W. den Herder, W. Hermsen, L. Kuiper \\ SRON-Leiden, P.O. Box 9504, 2300 RA Leiden, The Netherlands \\ A. Connors, R.M. Kippen, M. McConnell, J. Ryan \\ University of New Hampshire, Durham NH 03824, USA
}

\begin{abstract}
During the first year of operation, the COMPTEL instrument on board the Compton Gamma Ray Observatory detected $22 \gamma$-ray bursts within its field of view. Spectra and time histories for the strongest 7 of these bursts have been obtained from both the main instrument $(0.75-30 \mathrm{MeV})$ and the burst modules (0.1-10 MeV). The deconvolved photon spectra for the majority of bursts are fit by a single power law model with spectral index between -1.6 and -2.8 . One strong burst, GRB 910814, exhibited significant curvature and could not be fit by a single power law model. A broken power law model with a break in slope at $\sim 2 \mathrm{MeV}$ is a good fit to the time averaged spectrum of this burst. There is evidence, at the $2.8 \sigma$ level, for a change in the break energy of GRB 910814, from above $2 \mathrm{MeV}$ to below $1 \mathrm{MeV}$ during the first $9 \mathrm{~s}$ of the burst.
\end{abstract}

\section{INTRODUCTION}

COMPTEL is an imaging $\gamma$-ray telescope on board the Compton Gamma Ray Observatory (CGRO) operating in the energy range $0.75-30 \mathrm{MeV}$. The instrument consists of an upper layer of seven detectors (total area $4188 \mathrm{~cm}^{2}$ ), made from the low- $Z$ liquid scintillator material NE213A along with a lower layer of fourteen NaI cells (total area $8620 \mathrm{~cm}^{2}$ ). Two independent modes of operation are employed by COMPTEL for the detection of $\gamma$-ray bursts.

The "double scatter" (or "telescope") mode, which is the normal imaging mode of the telescope ${ }^{1}$, is used to produce images, spectra and time histories of bursts which occur within the field of view (FOV).

The "single detector" (or "burst") mode ${ }^{2}$ is triggered upon receipt of a signal from CGRO-BATSE that a burst has commenced. In this instance, two of the lower NaI detectors (called D2-7 and D2-14) accumulate 6 high time resolution ('burst') spectra for an integration time of $0.5 \mathrm{~s}$, followed by up to 255 'tail' spectra, each of $6 \mathrm{~s}$ integration time. Thereafter the burst modules return to 'background mode', integrating spectra for $100 \mathrm{~s}$ and awaiting the next BATSE trigger.

* Also: Astronomical Institute, University of Amsterdam, Kruislaan 403, NL-1098 SJ, Amsterdam, The Netherlands 


\section{Spectral Properties of Gamma-Ray Bursts}

The burst modules (each with a detecting area of $615 \mathrm{~cm}^{2}$ ) operate in overlapping energy regions. The low range detector (D2-14) is sensitive between $0.1-1.3 \mathrm{MeV}$ while the high range module (D2-7) covers the energy interval $0.4-10 \mathrm{MeV}$ (122 and 128 energy channels per module respectively). The spectral resolution is $9.6 \%$ at $0.5 \mathrm{MeV}$ and $7.0 \%$ at $1.5 \mathrm{MeV}$.

This paper presents a summary of spectroscopic results from the burst module data of $\gamma$-ray bursts which were detected in the FOV of the COMPTEL instrument in the first year of operation.

OBSERVATIONS

\begin{tabular}{|ccccc|}
\hline \multicolumn{5}{|c|}{ Table I Bright COMPTEL FOV Bursts } \\
\hline BATSE & Date & Seconds (UT) & Zenith & Fluence $(>0.6 \mathrm{MeV})$ \\
\hline Trigger & & & (Degrees) & $\left(\right.$ ergs cm $\left.{ }^{-2}\right)$ \\
\hline 109 & 910425 & 2265.75 & 44.8 & $5.5 \times 10^{-5}$ \\
143 & 910503 & 25452.7 & 23.0 & $1.5 \times 10^{-4}$ \\
249 & 910601 & 69734.55 & 8.0 & $3.5 \times 10^{-5}$ \\
451 & 910627 & 16157.78 & 10.0 & $2.6 \times 10^{-6}$ \\
503 & 910709 & 41602.13 & 36.7 & $2.8 \times 10^{-6}$ \\
678 & 910814 & 69273.04 & 29.2 & $1.3 \times 10^{-4}$ \\
1085 & 911118 & 68258.06 & 35.7 & $6.6 \times 10^{-6}$ \\
\hline
\end{tabular}

The BATSE all-sky monitors detected $\sim 300$ cosmic $\gamma$-ray bursts between April 171991 and April 16 1992. The COMPTEL burst detectors (modules D27 and D2-14) are, in principle, $4 \pi$ sensitive. In practice, however, their field of view is obstructed by a considerable amount of mass from both COMPTEL itself and the rest of the CGRO spacecraft. Only bursts with a zenith angle less than $45^{\circ}$ are considered in this paper because the sensitivity of the instrument in the forward direction is high and relatively uniform so the same detector response matrix may be used in the spectral deconvolution of these events. COMPTEL detected 22 events within its FOV during the first year of the CGRO mission, of which 7 were bright enough to be subjected to detailed spectral analysis ${ }^{3}$ (see Table I).

\section{RESULTS}

Single power-law, broken power-law (BPL) and optically thin thermal bremsstrahlung (OTTB) functions were used as trial photon models. The latter two were only applied when a single power law resulted in an unacceptable fit. The single power law model is of the form $\mathbf{a} \times \mathrm{E}(\mathrm{MeV})^{-\alpha}$, where $\mathbf{a}$ is the normalisation, or flux at $1 \mathrm{MeV}$ (in photons $\left./\left(\mathrm{cm}^{2} \mathrm{~s} \mathrm{MeV}\right)\right)$. The low range module (D2-14) was switched off between May 251991 and May 13 1992, so low range data are only available for GRB 910425 and 910503 . The results of the spectral 
L. O. Hanlon et al. 277

fitting using a power law model are shown in Table II.

\begin{tabular}{|cccccc|}
\hline \multicolumn{5}{|c|}{ Table II: Results of spectral fitting } \\
\hline Date & Duration & $0.3-1.3 \mathrm{MeV}$ & \multicolumn{2}{c|}{$0.6-10 \mathrm{MeV}$} \\
\hline & (sec) & $\alpha$ & $\chi^{2} / \nu$ & $\alpha$ & $\chi^{2} / \nu$ \\
\hline 910425 & 39 & $2.45 \pm 0.23$ & $28 / 38$ & $2.1 \pm 0.23$ & $13 / 15$ \\
910503 & 57 & $2.03 \pm 0.09$ & $99 / 81$ & $2.12 \pm 0.09$ & $39 / 36$ \\
910601 & 33 & - & - & $2.82 \pm 0.27$ & $9.5 / 10$ \\
910627 & 2 & - & - & $1.6_{-0.8}^{+1.1}$ & - \\
910709 & 0.5 & - & - & $2.0 \pm 0.42$ & $7 / 5$ \\
910814 & 33 & - & - & $2.06 \pm 0.07$ & $134 / 38$ \\
911118 & 9 & - & - & $2.64_{-0.38}^{+0.56}$ & $7 / 6$ \\
\hline
\end{tabular}

\section{DISCUSSION}

The $\gamma$-ray bursts shown in Table II are adequately described by a single power law model over their entire duration, with the exception of GRB 910814 . The best fit to the single detector data in this case was obtained using a BPL or an OTTB model. Neither model is rejectable on the basis of $\chi^{2}$ statistics. The spectral break is confirmed by BATSE ${ }^{4}$ and SIGMA ${ }^{5}$. The best fit BPL photon spectrum, averaged over the entire $33 \mathrm{~s}$ duration of the event, is shown in Figure 1.

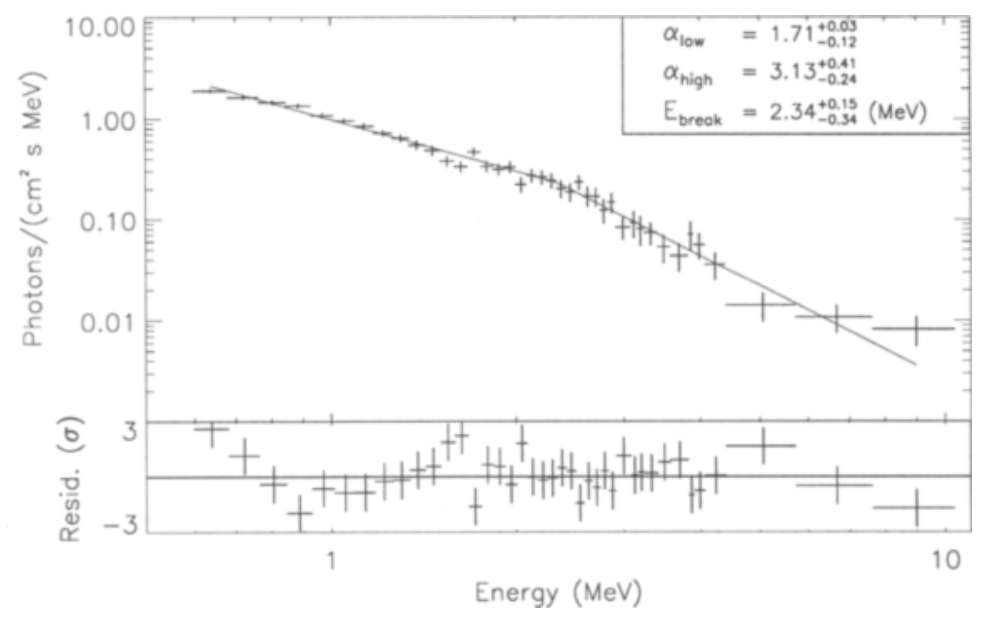

Figure 1. Photon spectrum of GRB 910814 derived from the best fit BPL model. $1 \sigma$ errors for one interesting parameter are given for the fit results. 
GRB 910814 was intense enough to be analysed in individual time intervals as shown in Figure 2. The intervals marked 1,2 and 3 represent $1 \mathrm{~s}$ time bins, while 4, 5, 6 and 7 are each $6 \mathrm{~s}$ in duration. These intervals have been used in the time-resolved spectral fitting of the burst mode spectra to investigate the spectral evolution in this burst.

The spectral curvature, requiring a BPL or OTTB model, is only significant during the first $9 \mathrm{~s}$ of the burst (intervals 1 to 4 ) after which time a single power law model is an acceptable fit. The deconvolved spectra for intervals 1 through 5 are shown in Figure 3. The variation in break energy, from above 2 $\mathrm{MeV}$ to below $1 \mathrm{MeV}$ during the first $9 \mathrm{~s}$ of the burst is significant at the $2.8 \sigma$ level.

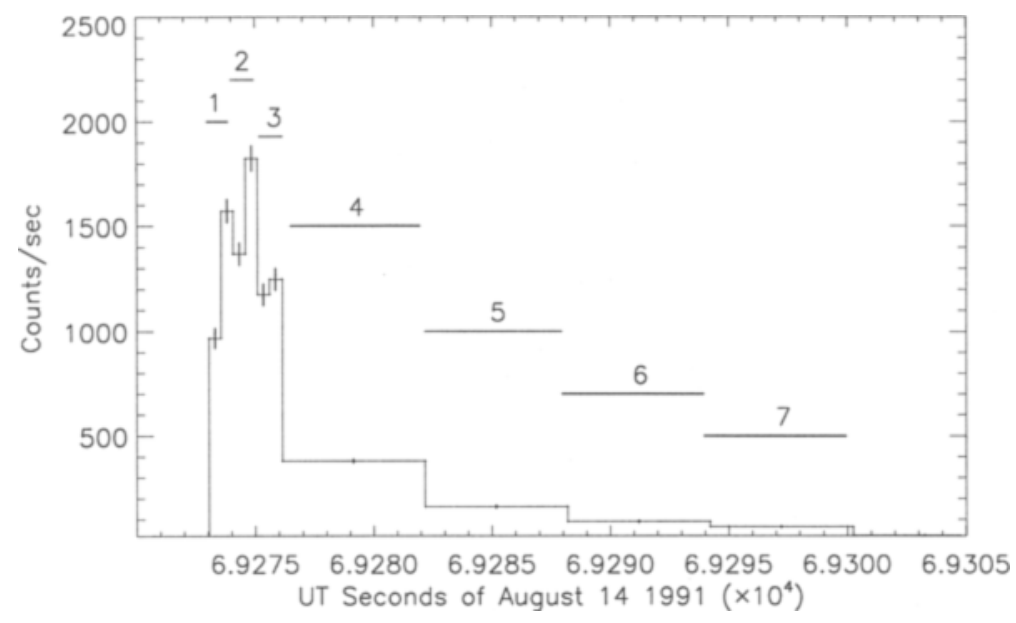

Figure 2. Background subtracted time history of GRB 910814 (0.6$10 \mathrm{MeV}$ ) showing time intervals used in spectral deconvolution

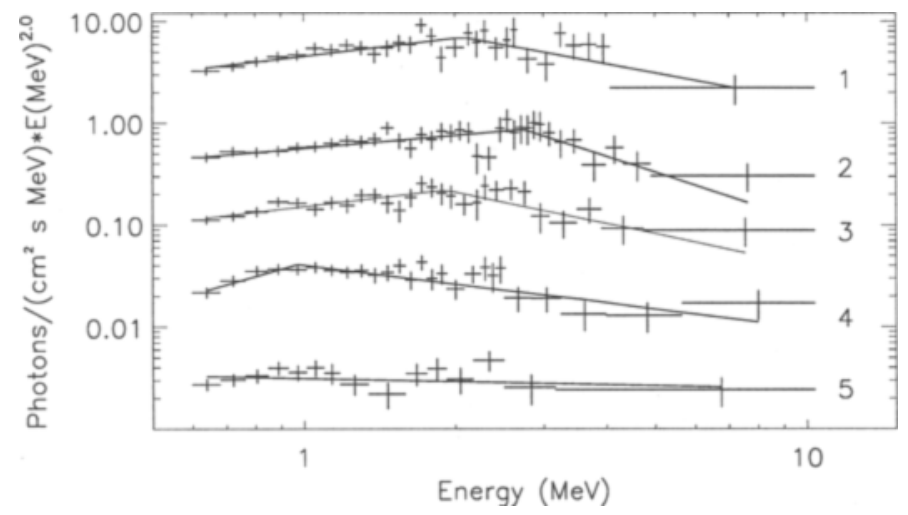

Figure 3. Photon spectra for time intervals 1-5 of GRB 910814

The time resolved spectral analysis of GRB 910814 leads to the possibility 
that the spectral break energy decreases in the course of the burst, such that after the first $9 \mathrm{~s}$, it has shifted to below the energy range of the instrument.

The EGRET data ${ }^{6}$ from this burst support this conclusion, since for the first $7 \mathrm{~s}$ of the burst, a single power law fits their data only if the first few points $(\sim 2 \mathrm{MeV})$ are not included in the fit. In subsequent time intervals, a single power law fits the data well over the whole energy range. Thus, the combined COMPTEL and EGRET results provide clear evidence for a time dependent spectral break at $\mathrm{MeV}$ energies in this $\gamma$-ray burst.

\section{CONCLUSIONS}

The majority of bright GRBs observed by COMPTEL had single power law spectra, with $1.6 \leq \alpha \leq 2.82$. Only one of the seven showed definite curvature, unlike the BATSE sample ${ }^{4}$ in which roughly a quarter of bursts exhibited curvature. These are not incompatible statistics, since some of the breaks observed by BATSE are below the COMPTEL energy range (e.g. GRB 910601 and GRB 910709). One bright burst, GRB 910814, exhibited a spectral turnover at $\sim 2 \mathrm{MeV}$ which is confirmed by other experiments. There is evidence in the COMPTEL data, supported by EGRET observations, that the spectral break energy of this burst is time dependent.

\section{ACKNOWLEDGEMENTS}

L.H. gratefully acknowledges an ESA fellowship. This research was supported in part by the Deutsche Agentur für Raumfahrtangelegenheiten (DARA) under the grant 50 QV 90968.

\section{REFERENCES}

1. Schönfelder, V., et al, Ap. J. Suppl. 86, 629 (1993).

2. Winkler, C., et al, Adv. Sp. Res. 6, 113 (1986).

3. Hanlon, L.O., et al, Accepted for publication in A\&A , (1993).

4. Schaefer, B.E., et al, Ap. J. 393, L51 (1992).

5. Pelaez, F., et al, Proceedings of INTEGRAL Workshop, (1993).

6. Kwok, P.W., et al, Compton Symposium (AIP 280, 1993), p. 855. 